\title{
Maternal gastric carcinoma with metastasis to the placenta: A case report
}

\author{
YUXUAN CHEN", YAO LI*, HONG WANG, JUNLI LU, MULAN JIN and ZHENYU ZHANG \\ Department of Obstetrics and Gynecology, Beijing Chaoyang Hospital, Affiliated Hospital of \\ Capital Medical University, Beijing 100020, P.R. China
}

Received December 27, 2013; Accepted May 29, 2014

DOI: $10.3892 / \mathrm{ol} .2014 .2529$

\begin{abstract}
In the current report a case of maternal gastric carcinoma with metastasis to the placenta is presented. Gastric cancer is a worldwide health issue, which accounts for $\sim 8.6 \%$ of newly diagnosed cancer cases annually. However, the simultaneous occurrence of gastric cancer and pregnancy is rare; to date, only $0.1 \%$ of all cases of gastric cancer occur during pregnancy. Furthermore, among all cases of gestational gastric cancer, only six cases have been reported to exhibit metastasis to the placenta or fetus. In the current study another such case is presented. Based on the results of previous studies, further discussion regarding the diagnosis and prognosis of gastric cancer during pregnancy in the mother and fetus is presented.
\end{abstract}

\section{Introduction}

Gastric cancer presents a worldwide health issue, which accounts for $\sim 934,000$ (8.6\%) of newly diagnosed cancer cases and 700,349 mortalities annually. Almost two-thirds of cases occur in Eastern Europe, South America and Asia, with $42 \%$ of cases occurring in China alone (1). The mean age of gastric carcinoma patients is 60 years, with a relatively infrequent occurrence in individuals aged $<40$ years. In addition, gastric cancer is more common in males than females, with a ratio of 1.7:1 (2). Only $0.1 \%$ of all cases of gastric carcinoma occur during pregnancy (3). In Japan, the rate of gastric cancer during pregnancy is reported to be just $0.016 \%$ (4); however, in China, it exhibits a frequency of $0.67 \%$, which is significantly higher than that of other countries (5). The diagnosis of this type of cancer is difficult due to the high frequency of benign gastrointestinal symptoms

Correspondence to: Mr. Zhenyu Zhang, Department of Obstetrics and Gynecology, Bejing Chaoyang Hospital, Affiliated Hospital of Capital Medical University, 8 Baijiazhuang Road, Beijing 100020, P.R. China

E-mail: ooozhenyu@126.com

*Contributed equally

Key words: gastric cancer, pregnancy, metastasis, placenta that are presented during normal pregnancies, which results in a poor prognosis, resulting in $88 \%$ of females succumbing to the disease within one year (6).

Gastric cancer metastasis invade the surrounding tissues and distant organs, affecting cancer cell motility, intravasation, transit in the blood or lymph and extravasation. Friedreich (7) reported the first case of carcinoma metastasis to the fetus in 1866; however, metastasis to the placenta or fetus is extremely rare with $<100$ cases reported (8). The most common among these are malignant melanomas, accounting for $30 \%$ of cases. Lung, hematological and breast malignancies are the second most common malignancies involved and gastric cancer accounts for $<5 \%$ of the cases reported $(3,9)$. To date, to the best of our knowledge, only six cases of metastasis from gastric cancer to the placenta or fetus have been reported. In the current study the seventh case is presented, which was the first case identified in China. The patient provided written informed consent.

\section{Case report}

A 35-year-old female (gravida, 2; para, 0) was admitted to Beijing Chaoyang Hospital (Beijing, China) at 34 weeks of gestation and was diagnosed with preeclampsia due to high blood pressure and a recent occurrence of proteinuria. The patient experienced occasional nausea and epigastric pain during the second trimester, however, did not receive adequate medical treatment.

Physical examination revealed that the uterus was normal for the gestational stage, without abdominal tenderness or peritoneal symptoms. In addition, the generalized superficial lymph nodes were found to be of normal size.

Laboratory test results revealed a white blood cell count of $13.94 \times 10^{9}$ cells $/ 1$, a red blood cell count of $2.86 \times 10^{12}$ cells $/ 1$, a hemoglobin level of $94 \mathrm{~g} / 1$, a platelet count of $125 \times 10^{9}$ cells $/ 1$, a D-Dimer level of $22.55 \mathrm{mg} / \mathrm{l}$, a fasting blood glucose (Fbg) level of $121.1 \mathrm{mg} / \mathrm{dl}$, aspartate aminotransferase levels of $14 \mathrm{U} / \mathrm{l}$, alanine aminotransferase levels of $13 \mathrm{U} / 1$ and albumin levels of $27.6 \mathrm{~g} / 1$.

An abdominal ultrasound revealed a 34-week single pregnancy. Two days later the patient developed a coagulation disorder, which resulted in a decrease in serum Fbg to $94.3 \mathrm{mg} / \mathrm{dl}$. An emergency cesarean section under spinal anesthesia was performed. The surgery revealed chylous ascites $(100 \mathrm{ml})$ and the placenta was pathologically analyzed. A premature female infant (weight, 2,210 g) with an Apgar 

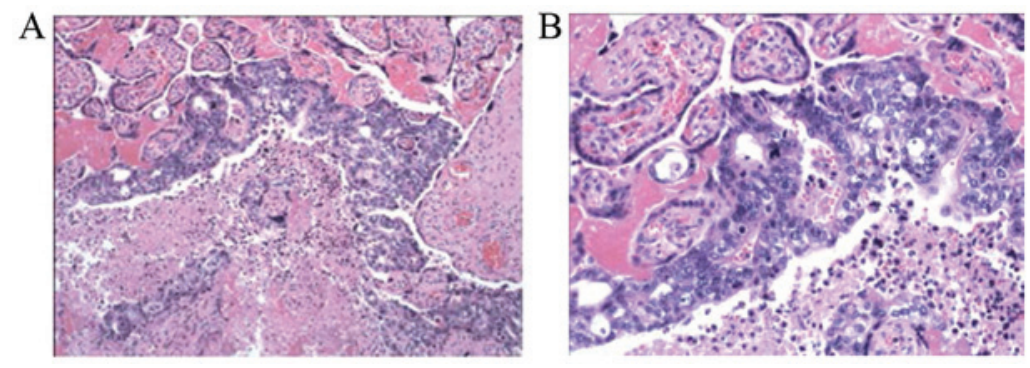

Figure 1. Microscopic examination of the placenta revealed gastric primary malignant cells infiltrating the intervillous space and villous. (A) Magnification x100. (B) Magnification, $\mathrm{x} 400$.

score (10) of 10 at 1,5 and 10 min, respectively, was delivered in a stable condition and was transferred to the neonatal intensive care unit of Beijing Chaoyang Hospital.

Macroscopic examination of the placenta revealed no gross metastasis. However, microscopic analysis revealed gastric primary malignant cells within the intervillous space with villous invasion (Fig. 1). The umbilical cord and membranes appeared normal. Computed tomography scan revealed fundus thickening and enlarged retroperitoneal lymph nodes. Gastroendoscopy was performed and revealed linitis plastica with hypertrophic rugae and ulceration. In addition, a gastric biopsy revealed an intermediate differentiated gastric adenocarcinoma. The patient survived and has received chemotherapy. Furthermore, the infant has been reported to be well six months following delivery, with no evidence of fetal metastasis.

\section{Discussion}

There is often a delay in the diagnosis of gastric cancer during pregnancy as the digestive symptoms are common and commonly considered to be a benign response that is induced by the pregnancy itself. This delay in diagnosis results in a poor prognosis resulting in $88 \%$ of females succumbing to the disease within one year (6).

Of the 92 cases of gastric cancer reviewed by Jaspers et al (11), almost all were found to be at an advanced stage of gastric cancer with only two cases diagnosed with early stage cancer. Early gastric cancer is difficult to detect during pregnancy, as the digestive symptoms of pregnancy, including nausea and vomiting, are common and do not usually require medical attention. Yoshida et al (4) reported a case of a patient with vomiting and epigastric pain in the second trimester. The obstetricians diagnosed the patient with early stage gastric cancer, which may have facilitated a favorable prognosis for the mother and infant. Thus, patients with unusual digestive symptoms subsequent to the first trimester require examination. Gastroendoscopy has been identified as an effective method for the detection of early gastric cancer and is regarded as a relatively safe procedure that may be performed, when clinically required, during gestation $(12,13)$.

Gastric carcinoma diagnosed in pregnancy is rare, occurring in only $0.1 \%$ of all cases of gastric carcinoma. Maternal malignancy with metastasis to the placenta or fetus is extremely rare with $<100$ cases reported, of which only $\sim 20 \%$ cases were reported to exhibit fetal metastasis (3). Gastric carcinoma accounts for $<5 \%$ of the reported cases. The majority of the reported cases were identified in the intervillous space without villous invasion; villous invasion is a significant risk factor for fetal involvement, which was present in the current case. Previous studies have recommended that a close follow-up of the healthy infant should be performed, including a physical examination, chest X-ray and liver function tests every six-months for the first two years of life. For cases exhibiting placental and fetal metastasis, further immunohistochemical examination of the mother and fetus is also advised (12).

In conclusion, the potential of gastric cancer during pregnancy must not be ignored, particularly in females exhibiting unusual digestive symptoms. Further investigation must be performed promptly for such patients, as early detection and intervention are essential for improving the prognosis of the mother and fetus. Histopathological examination of the placenta with precise examination of the intravillous spaces and villous is considered to be essential in every case of malignancy during pregnancy.

\section{References}

1. Power DG, Kelsen DP and Shah MA: Advanced gastric cancer - slow but steady progress. Cancer Treat Rev 36: 384-392, 2010.

2. Parkin DM, Pisani P and Ferlay J: Estimates of the worldwide incidence of eighteen major cancers in 1985. Int J Cancer 54: 594-606, 1993.

3. Khatib F, Shaya M and Samueloff A: Gastric carcinoma with metastasis to the placenta and amniotic fluid: case report and review of the literature. Eur J Obstet Gynecol Reprod Biol 107: 208-209, 2003.

4. Yoshida M, Matsuda $\mathrm{H}$ and Furuya K: Successful treatment of gastric cancer in pregnancy. Taiwan J Obstet Gynecol 48: 282-285, 2009.

5. Nv ZH: Clinical characteristics of gastric cancer during pregnancy and the reasons for misdiagnosis. Zhonghua Fu Chan Ke Za Zhi 11: 701-702, 2003 (In Chinese).

6. Hirabayashi M, Ueo H, Okudaira Y, et al: Early gastric cancer and a concomitant pregnancy. Am Surg 53: 730-732, 1987.

7. Friedreich N: Review of the pathology of cancer. Virchows Arch Path Anat 36: 465-482, 1866 (In German).

8. Baker AM, Haeri S, Shafer A and Moldenhauer JS: Maternal gastric carcinoma metastatic to the placenta. Eur J Obstet Gynecol Reprod Biol 153: 225-226, 2010.

9. Al-Adnani M, Kiho L and Scheimberg I: Maternal pancreatic carcinoma metastatic to the placenta: a case report and literature review. Pediatr Dev Pathol 10: 61-65, 2007.

10. Hacker NF, Gambone JC and Hobel CJ: Hacker \& Moore's Essentials of Obstetrics and Gynecology. 5th edition. Saunders, Philadelphia, PA, pp115-116, 2009.

11. Jaspers VK, Gillessen A and Quakernack K: Gastric cancer in pregnancy: do pregnancy, age or female sex alter the prognosis? Case reports and review. Eur J Obstet Gynecol Reprod Biol 87: 13-22, 1999

12. Pentheroudakis $\mathrm{G}$ and Pavlidis $\mathrm{N}$ : Cancer and pregnancy: poena magna, not anymore. Eur J Cancer 42: 126-140, 2006.

13. Offerhaus GJ, Tersmette AC, Giardiello FM, et al: Evaluation of endoscopy for early detection of gastric-stump cancer. Lancet 340: 33-35, 1992. 